

\section{Modernidad expandida. Perú en el libro ilustrado argentino (1920-1930)}

Rodrizo Gatierre Viñales Maversidad de Grantada

Il presente estudio, basado en investigaciones iniciadas hace ya una década, centra la atención en los contactos artísticos entre Perú y la Argentina, tomando como referente especifico a la ilustración de libros. Se situa temporaimente en un momento crucial para el arte latinoamericano como es el de la consolidación de la modernidad y el arraigo de las corrientes de vanguardia, en cuyos escenarios sera decisivala presencia dei diseño grafico. A lo largo del ensayo nos referimos justemente al libro illustrado como factor de modernidad, antes de adontrarnos on el núcleo central de la cuestión como es la presencia de literatos Y artistas peruanos en el ámbito del libro llustrado argentino, con particular atención al arequipeño Julis Málaga Grenet y a una de las temáticas emblematicas del vinculo entre ambos paises: el incaismo como expresión de vanguardia con raices

Perú / Argentina / Década de 1920/ Modernidad / Vanguardia / Diseña gráfico / Libros ilustrados / Julio Málaga Grenet / Prehispanismo / Incaismo

The present study, based cn research started a decade ago, focuses on artistic contacts between Peru and Argentina, on the specific reference to the illustration of books. It is located temporarily at a crucial moment for Latin American art as is the consclidation of modernity and establishment of avant-garde currents, whose scienarios will be decisives in the presence of graphic design. Throughout the essay we mean precisely the illustrated book as a factor of madernity, before we delvo into the core of the issue as the presence of Peruvian writers and artists in the fieid of Argentine's illustrated book, with particular attention to Ju is Malaga Grenet and one of the emblematic thematic link between the two countries: the incaism as an expression of art with roots.

Peru / Argentina / 1920s / Modernity / Avant-garde / Graphic Design / Picture books / Julio Málaga Grenet / Prehispanism / Incaism

1. Introdacción. El libro ilustrado como factor de modernidad

Al echar la vista sobre las "historias del arte" esciltas en Latinoamérica durante el siglo XX salta a la vista que las mismas estan sustentadas casi exclusivamente en pintura y escultura. con alguna tibia incursión en grabado y dibujo fsobre todo en los últimos listros y con casi nada tespecto de disefio gráfico, caricatura o artes decorativas, testimontios que, cuando han recibido algun tratamiento. este ha sido mas bien marginal y cercano a bo artecdócico. Esta omisión se vuelve más sangrante cuando se asume como tema de análisis el origen y consolidación de lo que llamamos "modemidad", que podriamos situar entre finales del XIX y hasta los años 20 , decada esta ultima ya marcada por una cronstancia ineludible 


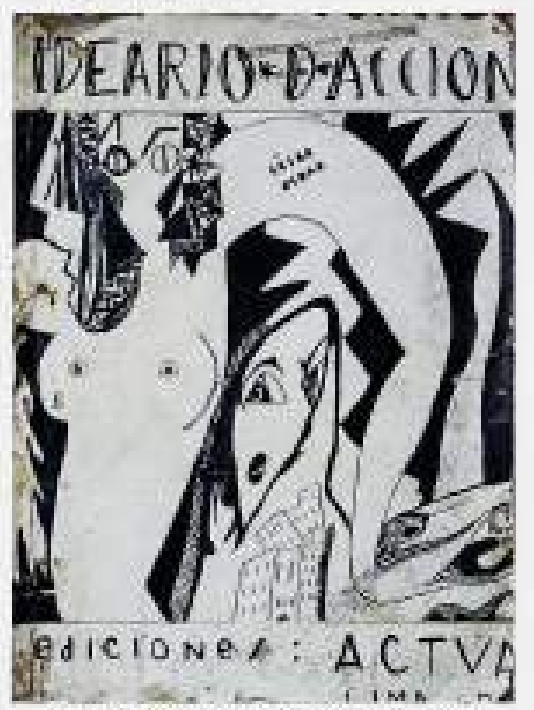

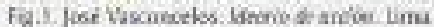

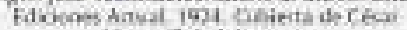
Mons Cul del auter

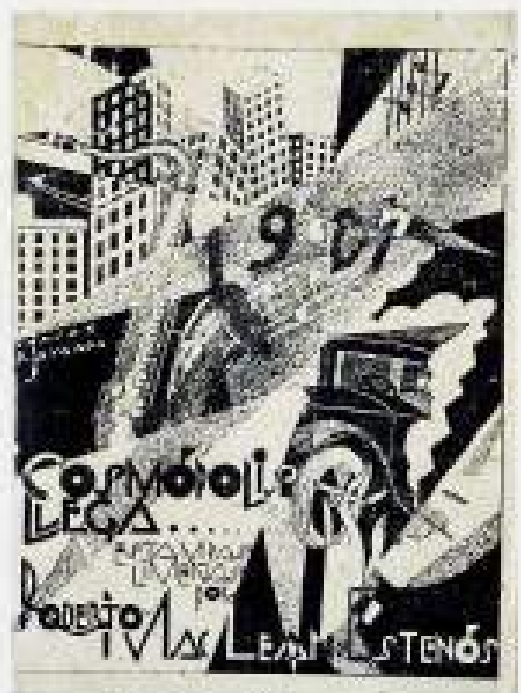

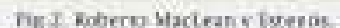

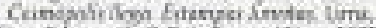

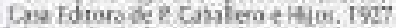
Cutretade Carbs Ragade cot.delauteri para kas historiadares como es la descratcutzacicin de las artes como praxes y el proceso de integracion de las mismas, confommandose àmbitos donde arquirectos trabajaron can escultores, pintores con gente de tuatro, o ilustradorics con literatos, en un cruzamiento disciplinazio de gran vitalidad, en donde los creadores supieron incursionar en generos a priori ajenos.

Cuando se analiza a fondo tese decenio, ise advierte que. en muchas ocasiones, los artistas se mostraban mas abelantados en sus dibujos y grabados que en sus propias careas cono pintores. las estampas, revistas, libres, partituras 0 carteles fungian come verdaderos laboraboriosy. por lo general, resultaron más permesbles a la innovación. gozando además de mayor capsicidad en to que atañe a la conformacion del gustis popular. Fueron áreas experimentales esenciales para entender la modemidad, en la que es ia. sosiayable la importancia que supuso ef contacto con literatos y editores. con los que trabajarian codo a codo, ampliando sus horizontes. Las libros ilustrados son el resil tado de ese proceso. Como dice Juan Mancel Bonet:

meen la época de la que hablamos, los iibros estan bechos a la vez por el poeta y par el gráfico... El libro se pienss como una totalidad; to escrite y b gráfico tienen la misma importancia y a veces ef poeta ni siquiera precisa un ilustrador, el misme acude a esa parte. '.

Los avances del conocimiento en cuarte a rstos materiales que ticnen como sizno la multiplicidad y el soporte papel, nos han obligado a reescribir aspectos de nuestras historias del arte contemporaneo y, como consectsencia, a adaptar recorridos y discursos en museos y exposiciones temporahes, a veces al puato de que en estas últimas, cuando no se exhiben vitrinas con material grafica y documental, pademos tener la sensación de estai ante maestras sospechosas de no cosllevar urat profunda tarea de imestigación detras. En otras palabras, que nos hemos acostunbrado a la necesidad de visualizar esos materiales. Por suerte esto va roda vez mis cn alza, y por poner el ejemplo más reciente en Lima mencisnaremos la exposición de Jese Sabogal inatgurada en el MALI en julio de 20313:

Las tlustraciones pain libros y revistas constituyen una de las reservas

1 Itoptone, 2011 
artisticas mis importantes del perio: do... Prejaicios muy arraigados han impedido que sean apreciadas en su fusto valos, frente 2 otras obras con. fiadas a moportes considerados, sin jastificación, más nobles que el frágil papel. Como consecuencia de esta falta de incerés, artistas que brillaron con toda intensidad en sa época, son practicamente desconocidos por las generaciones actuales!

$O$ como escribio el mexicano Xavier Moyssen:

E papel desempenado por la literatura en la evolución del arte moderno y rusy en especial el que corresponde al libro iluscrado, no ha recibido la atención que merece... Los libros son bienes poco frecuentes en las colecciones museisticas, que suelen conar con und estructura de departamentos tradicional y un público acostumbrado a encontrat pinturas y esculturas en las salas de exposiciont,

Por ello es de aplaudir la tendencia cada vez más consolidada de pronnover publicacicones y exposiciones que tienen a libros y revistas ilustradas no ya como complementos sine como protagonistas principakes. No vamos a extendemos anui en hacer un listado de casos, pero sí mencionaremos, a manera de muestra, la exposición la von. guardia apiicade (1890-1950) llevada a cabo en la madrilena Fundacón fuan March durante 2012 , hermanando las sobresalientes colecciones del estadounidense Menill C. Berman y det espanol Jase Maria Laftuente

En esta muestia en lo que toca at Perù, se exhibió un ejemplar de Quinnica dei espuritio de Alberto Hidalso, Jibro publicado en Buenos Aires en 1923 yen el que el autor uisiós sus fikcetas de escritory tipógrafo, y en la que no hubieran desentonado para nada excelentes muessas de la vanguardia peruana como la muy temprana y poscubista cu. bierta de Cesar Moro para ldecrio de occión (1924) de fosé vasconcelos (Fig.11, la neofurutista de Carlos Raygada para Casmópolis Hegr 11927 de Roberto Maclean y Estenós (fig. 2). La xilogralica composición de Emilia Coybuni para los 5 metros

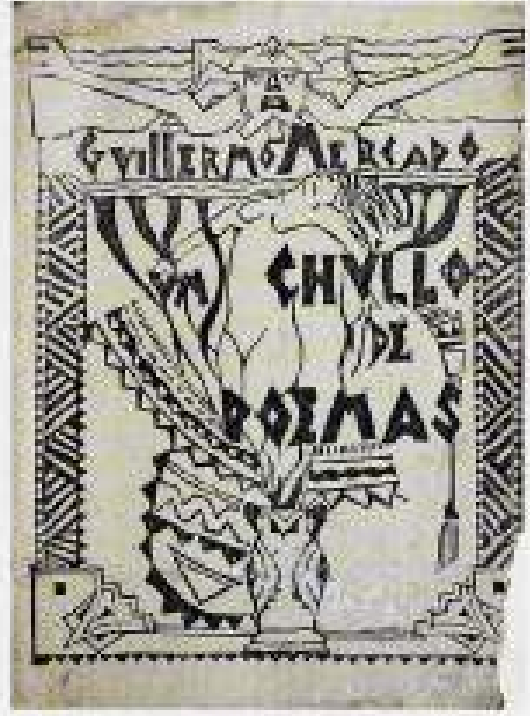

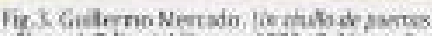
Siciani. Edisonal kurme. 1928. Cubicra 60

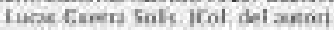

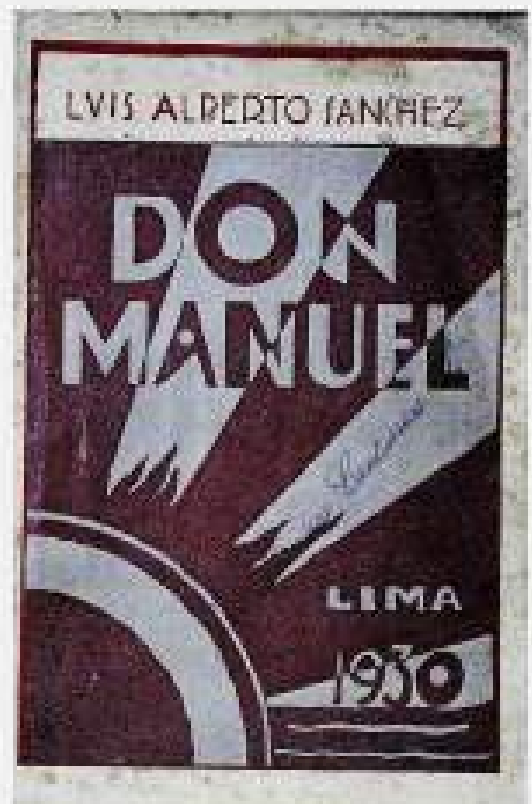

Fig. 4. Lin Abenn Sineter. Dan Maver. Uirn. Aibetia Flancisz Dectifica. 1930 Cutiera de pous Sabagil iCnil del watart. 


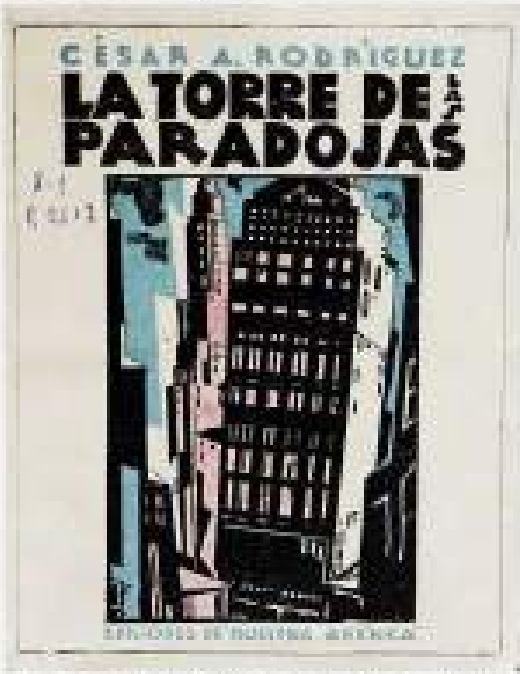

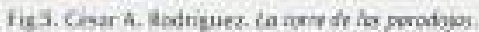

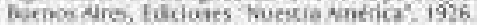

Cidresta de jase Bunnom. Ket delautici

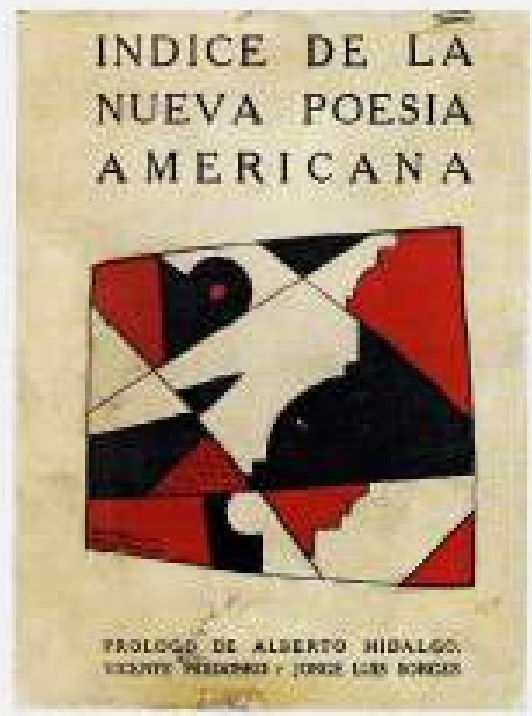

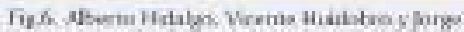

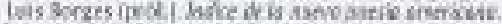

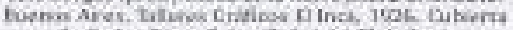

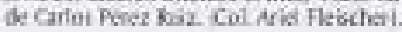

de poumas de Carlos Oquendo de Amat, ha incais ta de Lucas Guerna Solis para Un cheilly de poerties (1928) de Caillermo Mercado (Fig.3), o inclusive la de tintes deco que jose Sabogal hace para Dom Manzed (1930). Iibro que Lus Aberto Sinchez dedica z Manuel Goazalez Prada (Fig 4).

En et ambito latinoanericano, podemos señalat como el testuerzo mas importante dado en este campo la exposición y litso México linsfrado, 1920-1950, coordinados por Salvador Albinana en 2010, que, desde st primera puesta en excena en el Museo Valesciano de la llustración y la Modemidad (MLIVIM), circulo por varias cisidades europeas y americanas hasta 2012 . En junio de 2013, bajo la curaduría de Riccardo Boglione. se inatigura en el Musea Nacional de Artes Visuales de Montevideo la muestra Wibrackir gro-

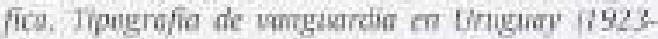
1936). En naessro caso personal, al momento de escribir estas lineas, tenemos en proceso de maquetación la obra titulada Libmer orgentimos. Plistracion y modemidad (1910-1936) que esperamos vea la laz a principios de 2014.

\section{Perí en el libro argentino. De literatos y artistas}

In 2009 publicamos en lima, con Dizabeth Kuon, Kamón Gutiérrez y Graciela Vinutales, el tibro Cuzco-Buenos Aires. Ruta de intulectualidod ame. ficana (IS000-1950), en cuyo procese de ithestigación advertimos la notoria importancia que habian tenido, en los intercambros producidos en ese eje geográfica, los lithros y las puhlicacio-

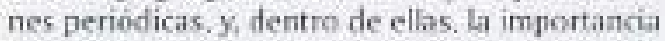
dada a las ilustraciones, mayoritariamente de sesgo prehispanista,

Esa sencación se confirmó y acentuó al acometer poco después el estudio sobre los libros argentinos mencionado, donde el numero de ejemplos clasificables dentro de ess linea fue ent aumento. determinando que la misma se convirtiera en un horizonte presente. En ese marco podemos situar, en la que atane a los lustradores y sas vinculos con los literatos, al arribo en 1909 del dibujante arequipeno Julio Malaga Grenet, y en $19: 1$ de kosé Sabogally el crítico Alfredo Cliabra Acosta Aralayal. A partir de los años 20 destacará fundamentalmente el pocta y antólo-

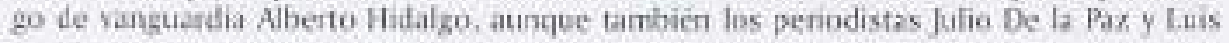

+ haon Arie y cerou, soos. 
Fernaín Cimeros, quien contó eespectivamente, para la primera y tercera edición de su Todo es omor [1923 y 1933], con el concurso de Mallaga Grenet y def argentiso Julio E. Payró, uno de los criticos de arte màs influyentes del pais y que en su juventud tuvo algunas incur. siones puntuales en el disemo grafico, Y podriamos extendernos territorialmente $y$ citar la presencia, en este caso en Uruguay, de juan Parra del kiego, casado con la poctisa uruguaya Blanca Luz Brum, a quien dedicaria, poco antes de su fallecimiento, su libro Blanca Luz (1925), con cubierta ilustrada por el montevideano Cesar Pesce Castro.

En 1924 se fundó en la capital argentina la editorial Nuestra América. con la intención de dar a conocer a escritores de todo el continente. Esta empresa que llegaria a editar una decena de litros, en su mayor parte ilustrados por un notable (y ohvidado) artissa de la varguandia argentina, Jose Bonani. Dus peruanos se desempeñaron como sepresentantes de la editocial en el exterior. Ventura Garcia Calderón en Paris y César Atahuelpa Rodriguez, en Arecuipa. Bonomi les il istraria respectivamente las cubiertas de Sonrisas de Paris (1925). con unz sintetica Torre Fillel. y la torr de las poradojas 11926. (Fig.5) en la que sobresalen altos edificios y rascicielos. Las cubiertas "pervanas". de Bonomi se completarian con la del libro Tiempos de la patrin siejo (1926) de Angelica Palma. geometrizando a través de tintas negras y rojas una escena historica acorde con los coirenidos.

Figura cencral fue Aberto Hidalgo, promotor, a mediados de los 20 . de una peńa literaria que se reunia los sábados en el Cafe "El Clobo", y que aglutinaba a varios escritores y artistas vinculados a otro de los órganos de la vanguardia atgentina, la revista Pron, entre ellos Jorge Luis Borges, Con éste y con el chileno Vicente Huidobro. Hidalgo editaria en 1926 el findice de la autur poesia anersuria con fantedstica cubierta poscubista de Carlos Pérez. Ruiz (Fig.6), artista perteneciente al mismo circulo. En cuestión de Libros, Hidalgo habia recurrido en algunas de sus obras a tipografias de avanzada como en Quiuira def espinitu (1923) o Simplismo (3925). En esos años cimentarfa una estrecha amistad coo el pintor vanguardista Enilio Pettorati-también amigo de lose Carlos Maraitezui-, quien le pintaria al menos tres retratos a Hidalgo, reproduciéndose uno de cllos en la cubierta de la edición privadia de éste, Diario de mi sentimienta $\{1937\}$.

Ya en Ics años 30, una última cubierta a citar fue la que el rosarino fulio Yanzo diseñó para el libro Nuevo arte (1934) del piusano Felipe Cossio del Pomar. Vanzo fue otro de los artistas de vanguardiz con amplia trovectoriar en la ilustración de libros, $y$ esta es su peimera andadura conocida en el arte del fotomentaje, combinando la fotografia con sus habituales composiciones geometricadas a base de Lanco, negro y rojo. Un ejemplar de este libro fue iachuido en la ya citada exposición Lo zangziordír apdicoda (1890-1950).

En to que se refiere a lustradores peruanos, quien adquirio mayor protagonisme dentro del ambito de Lu ilustracion argentina es sin duda Malaga Grenet, a quiea dedicaremos un apartado especifico. No obstante, aunque se trata de una acciòn indirecta, debernos mencionar también el rrabajo como llustrador de libros de José Sabogal. Si bien las obras a las que nos referimos fueron publicadas en Lima y Madrid enire 1919 y 1922, es decir entre su retomo de la Argentina y antes del viaje con Masia Wiesse a México en el que neorporard a la xilografía como lenguaje vinculado al diseno gráfico, no cabe duda que sus iniciales inclinaciones simbolistas estaban infludas por su contacto con el medio argentino. Luego de su llegada a buenos Aires en 191 i se vinculó a lat Academia de Bellas Artes, teniendo comto compasiero, entre otros, a Alfredo Guido, uno de los más notables artistas argenti. nos, que seria esencial en todo lo referente al diseño prehispanista a través de mobsiario y otras expresiones en el ámbito de las artes decorativas, ilustración en libros y revistas. estampas, murales y óleos". 


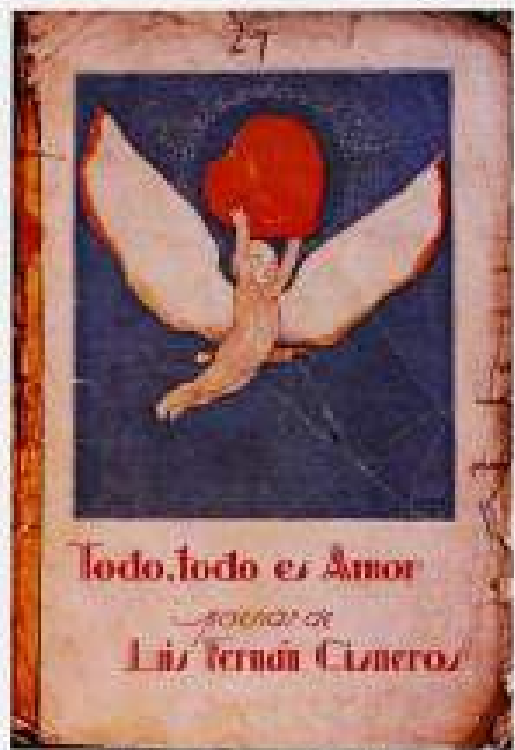

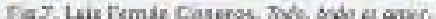

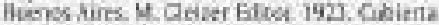

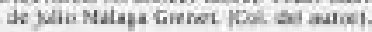

Tras la larga estancia en Telcara, entre 1913 y 1918, ano en que retoma a Buenos Aines y participa del "Primer Salon Nacional de Aztistas Independientes sin jurados y sin premios? Ilevada acaho en el salori costa, Sabongl tiene ocilsóin de visitar ones "Primer Salcan", el de Artes Decorativas, en el que justamente Alfredo Guides. junto a otro rosarina-José Gerbino, obtienen el primer premio por un Cofre de estilo incuivo. En ese salón se exhben numerosas propuestas de ilustracion para libros, mayoritariamente con rasgos simbolistac, del tijo de tas que, coiritidentemente, estäs invadiendo la producción editorial. Cuando Sabogal regresa al ano siguiente al Perú, trae consigo todo este bagaje, que indure el conocimiento de revistas como Nas Uitra, aparcida en 1916 como lujoso suplemen: to de la masiva Coros y caretas inuy en la linea de la limena bariedaderi En la misma, llustradores de renombere en la época como Gregorio Lojpex Naguil, jorge Larco, Rodolfo Franco o el citado Guido. dejan su huelda simbolista a lo Aubrey Beardsley, senda en la que también ilustran namerosisimos libros en esos años.

Aungue no se trata estrictamente de una "ilustración para libro", la inclusión por parte de Sabogal, ya en 1919. de un retrato del poeta Alberto Hidalgo en la cubierta du su libro Jardin Zoológico (se prohibe lo entrodo a hrs menores de ectad) publicado en Arequipa. nos habla a las claras de la atención que prestaba a ese tipo de labores. En 1920. Sabogal iniciara una estreclsa y efimera, pero trascendente, colaboración con el escritor Daniel Ruzo, a quien alustrara dos ediciones de su libro Asi hu cantada la naturalezo (1920), luego Modrigads (1921) y, finalmente El etrio de las lampanas (Madrid, 1922). Detrais de Sabogal vendrán vatios artistas lanzados, en afín modernizador, a la ilustración de libros en el Perü; por citar solo algunos casos mencionaremos a Carlos Quizpez Asín lantes de su ida a España en 1921), a Raúl Pro, Alejandro Gonzáles Trujillo, Victor Morey. Ratíl Vizcarta e inchusive a Emilio Harth-Terré, autor en 1924, un año antes de graduarse como arqui. tecto, de siatéricos dibujos para el poematio en francís en passant de su colega hector velarde.

\section{Julie Málaga Grenet y la ilustración de libros}

Dentro de estos escenarios de modernidad, como seîalamos con anterionidad, sobresalió el arequipeno Julio Málaga Grenet, guien habia triunfado con sus caricaturas durante la primera decada del XX en prestigiosos periodicos y tevistas limefios: Er tal sentido podemos destacar su acción en actualidades ise vincula a la misma en 1904). Monus y mononas. que dirigió junto a Leónidas Yerovi a partir de su creación en 1905, y Variedades, a partir de 1908. en la que seria remplazado dos acios después por Francisco González Gamarra. Fue considerado el mis sagaz de cuantos hicieron, mediante la plama, una lectura satinca de los sucesos de la politica nacional. 
En 19199, un ano antes del Centenario argentino, en la plenitud de su exito, y debido a una caricanura suya el semanario figaro, en el cual trabajaba, fue clausurado. Malaga Grenet tomb́s entonces la decisión de marcharse a Buenos Aires, donde se consolidó pronto, junto a su anigo Alejandro Sizio y a otros artistas del gremio, como uno de los ilustradores prín cipales de lavida social argentina, de su historia y sus circunstancias. Asf fo hizo en Carasy corrtos ide la que tras su llegada se convirtió en clirectó artistico) o. más adelante. en Plis Ibitra, Mando Arzentimen y 17 Hoger, organos de difusion masiva de alta circulacion.

Aun mediando una larga remporada en que regreso a lima, entre 1916 y 1921, para vincularse a la direccion del diario El Peni junta a Luis Fernan Eisnems, tarea que a la postre seria efumera, o para colaborar como dibujante en Excelsior o la revista Don Lanes, Málaga Grenet tería ya prestigio consolidado en la capital argentina doade a partir de 1921 se desemperiaria eomo director artistico del suplemento dominical del diario lo Nocín. De esos anos datan sus primeras ilustraciones para libros: Cantos de lo montala (1921) de fuan Carlos Dávalos. Tes relatos porfeños $(1922)$ de Arturo Cancela, Adriana Zumarán (1922) de Carlos Aherto Leumann. Patricio (1923) de Emesto Renan, y Toda, todo es ampr (1923) del citado Cisneros (Fig .7).

De 1923 data uno de sus más recordados traba. jos como dibujante publicitario. el cartel de la cerveza Quimes Cristal. Ese aho marcha hacia Nucva York, pasando por lima, En Norteamérica dara continuidad a esa labor cartelistica alcanzando gran exito, publicando elt varios diarios y revistas. En et ámbito def libro su accion mas relevante serain las cubiertas que realice para las ediciones neoyorquinas de Hugo Wast. publicadas pox Longmans Green \& Co., entre ellas Black Walley: A Rumance of the Argentine (1928). Stove desert 1928). Peace blassan (1929) y The strength of lovers (1930). También las de las dos ediciones det Almanaque de la mujer $(1929$ y 1930$)$ que se pubtican en thenos Aires, cuyas ilustraciores firma en Nueva Yock. En 1930 va a Furopa, visitando Portugal, España e instalandose en Paris. Publicara alli dibujos en Le rine y Fantasio además de ilastar \$ang gitante (1933) de Raymond Escholier publicado en la capital francesa, en edi-

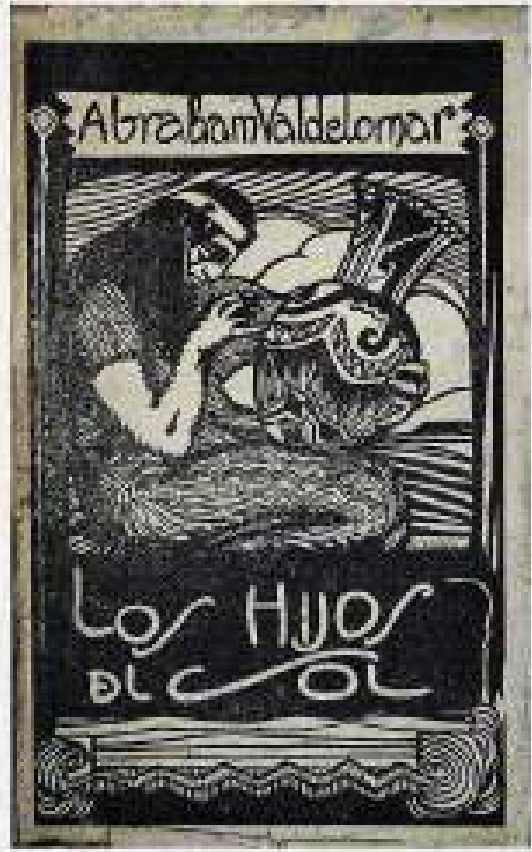

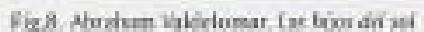

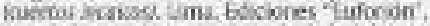
1921 Cuberto de hat Sabagal oc ol delauteri

ción reservada exclusivameate al cuerpo médico vinculado a los laboratorios. Deglaude, y en 1935 la tapa de Ciudad togica inowela hishovica periana) de Francisco A. loayza, lanzado en Barcelona por Maixci. En 1936 se produce su retomo a la Argentina. y en 1940 a su pals natal, radicándose en Limas. De este periodo podemos mencionar las ilustraciones que hace para Calles de Lima y meses dé ono (1943) de José Gálvez. Falleceria en 1963.

\section{El incaismo en la ilustración argentina. Una vanguartia enraizada}

Hace algumos anos escribía Alfonso Castrillón que "el incaismo puede ser definido como una tendencia plastica peruana que utiliza motivas ornamentales incaicos adaptados. 


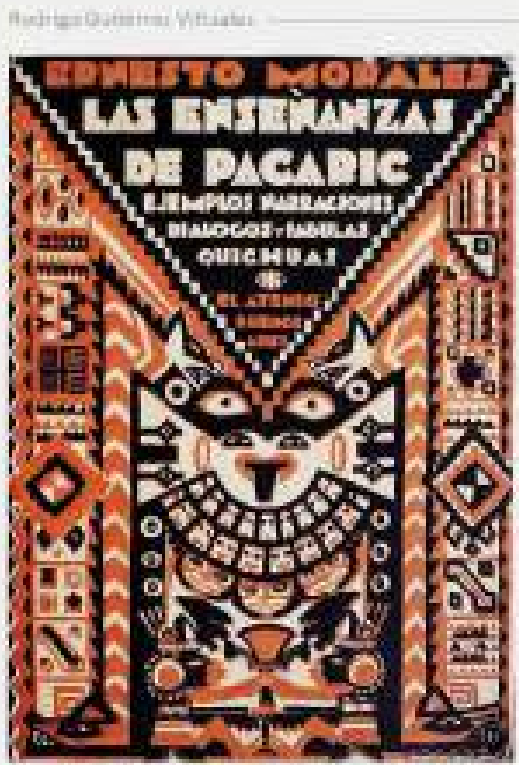

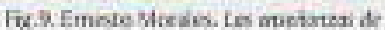

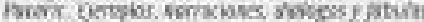
Fictuss Duems Nines. 'El Avenev. I929

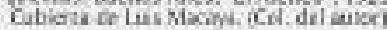

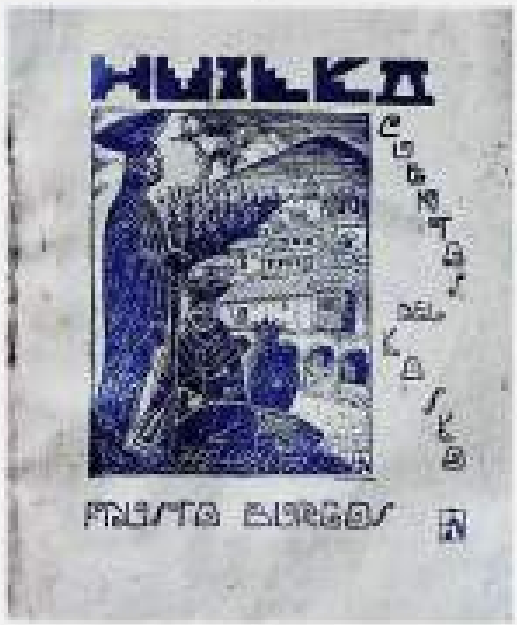

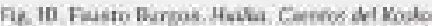

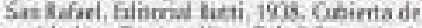

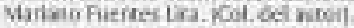

algunas veces, a los modelos occidentales y de inoculable coutenhto romántico por su anoranza de un munds arczidico ya supernda" Al incaismo", en el concierto latiooamericano. podriamos signarlo cormo un framento de แล concepto más amplio, el "prehispanismo". que en buema medida fue expresion artistica de yanguardia. Ha existido y existe una suerte de incomodidad historiogrifixa con respecto a estos restimonios por no ajustarse a los itineraios de avanzada tradicionalmente trazados, de seago europeo en su mayotia. Pero no en vano, uno de los mayores expertos en las vanguardias histuricas. Serge Fauchereas, incluye dentro de las mismas a varias vertientes americanas: estridentismo, muralismo, indigenismo, antropofagismo ${ }^{18}$,

De los personajes y medios vinculados habitual. mente con las vanguardias, varios y destacados son los que mostraton una intimz comanion con lo prehispánico. Para los dos paises que nos ocupan aqui, Argentima y Perzi. lo autóctono fungiob como una verdadera vanguadia con tradición: basta con pensar en los titulos de las que son quizá las más importantes revistas de vanguandia en ambos paises, Martiw Fierro y Amaurn, que re. curren a referentes venáculos en logar de-indinarse por fórmulas más estridentes

La fascinación que el arte prebispánico cauxol en muchos espiricus de vanguatdia es tangible. y en el caso argentino pxiriamos mencionar a Oiverio Girondo, compulsivo coleccionista de pezas precolombinas, o a Alberto Prebisch. autor éste de das recordadas confrontaciones visuales insertas en Mortin Fierno en 1925, en las que contraponía esculruras aztecas fboena y magnifical a obras de jos escultores Yrurtia (inalo) y José Llimona bésinnl, además de bacer referencias en algunas de sus criticas de arte con. temporàteo a lo genuino de aquelias creaciones preteritas", Estil "vanguardia enraizada" tuvo un amplio campo de acciòn en el diseño grafico, tanto en to que se refiere a la ilastración como a bo tipogrifico, ambito este en el que se consolidaron originales propuestas, netamente americanistas. En Peri el cjemplo de Elena lzcue, cuyo rescate lo considerames uno de los

\footnotetext{
9 Contalón, 2006: 37

10 Pincherein. 2010

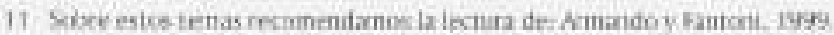


mayores que se hayan becho en of ímbito de las vanguardias latinoamericanas? is mas que esclarecedor.

Aun en su sesgo vanguardista, lejos estuwo el prehigpanismo de ser una expresióa "rupturista": sus formas estaban presentes ya en el simbolismo y en las proptestas del art animau. y servirian de puente anticipando la geometrización deco con la que luego se entroncaria. La eatrada al art deco proporciono a lo prehispánico un rumbo estético de avam. zida, permitiendo una estilizacion de los lenguajes indizenas, y el rescate de sus formas geométricas, las que se revisitan para descubrir en ellas formas modernas

Quienes primero se interesaron por estos objetos fueron los artistas y no los feóricos del arte, y por una razón fundamentalmente artistica, la posibilidad de poseer un repertorio de formas con siglos de experimentación al akcance de la mano, Los artistas curopeos, formados todos en el seno del academicismo y ef naturalismo, veian en los objetos primitlvos la concreción de aquellos priscipios significativos, abstractos, conceptuales y sintéticos que buscaban... En el camino de la abstracción y la sintesis de la forma los realizadores de formas primitivas les ilevalsan una incalculable ventaja'1?,

En ef libro periano fue, otra vez. José Sabogal uno de los primeros en aplicar los postu: Lados incaistas, haciéndolo tempranamente, en 1921, con las cabiertas de les bijas de! sol (1927). Lhro postumo de Ahraham Valdelomar (Fig.8) y de Mores artificinies de fose Choino. Entre las mis emblemáticas cubiertas en esta linea podriamos señalar la del ya referido Un chuilo de poentus (1928) de Guillermo Mercado, o la de E numo indio (1930) de José Lriel Garcia, con ornamentaciones y tipografia prehispanistas.

Lo incaista, tal como recogimos en auestro libro Cizco-Baenos Aires anteriomente citado. alcanzó fortune en la Argentina como ideologia y actitud estética. En el ámbito del libro dustrado tin ejemplo temprano es la cubiena que hoce Fidel De lucia para el "poema incaico" Los virgenes def sol $(1920)$ de Ataliva Herrera, libro que desarnolla su trama en el proceso de derrumbe del imperio incaico, con la traicion de Francisco Pizarro a Atahualpa y al aprisionamiento de este.

Siempre en la capital argentina. en 1923 se producirán dos hechos de relevancia para nuestro itinerario uno, el lanzamiento de los cuadernos varacochia que publican el escultor Gonzalo Leguizamóa Pondal y el arquitecto Alberto Gelly Cantilo, con el fin de crientar la ensenanza del arte decorativo en la Árgentina, tomando motivos de la región diaguita. calelraqui, considerados por ellos "de facil adaptación ea la decoración moderna"14. Otro. el arribo desde Cazco de la Companía Incaica dirigida por luis Valcárcel para hacer representaciones del drama quechea Olentar. El argentino Rodolfo franco haria el cartel publicitario $^{15}$ y colaboraría en la parte escenografica. Franco, uno de los artistas esenciales en el libro argentino de esos años, ilustraria a traves de una fantasia arọuitectónica incaista la tapa de otro Los hijos del sol, este de Arturo Capdevila, una epopera incaica, tratadir con rigor histórics. santo que al final incluia abundante bibliografia sobre los temas tratados.

En esos años finales de la decada del 20 otro ilustrador. el catalán radicado en la Argentina Luis Makaya, se convertirá els el más prolifico dentro del tópico, a través de la colabora. ción con dos literates locales inclinados a las temáticas indigenas con expecial atención a lo incaico, Ernesto Morales y Justo G. Dessein Merio. Para ambos, Macaya disenará tapas en Las que plasmará iconografias y tipografias prehispanistas que aleanzan en ocasiones

12. Majhif Y Witfanden, 1999

13 Ocarnon. $211 \leqslant 99-100$

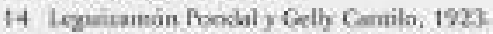

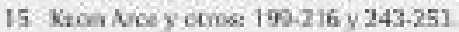


gran calidad como se aprecia en Leyeodas de Jndies (1928), 2as encenanzas de Pocanic (1929) (Fig 9) y fscudlos incaicns de Morales. o en Avides det sol 11929) y Aro incaica 11929 ) de Dessein Merla.

Sin embargo, ol literato argeatino màs volcado a los teras andinos dal Perü va a ser Fausta Burgos, radicado a finales de los años 20 en la localidad mendocina de Sas Rafael. Para la mayor parte de los libros que editó alli, baje el sello editorial Butti. requirió la colaboración de ilustradores, en su maycria de tranectoria reconocida como Antosio Bermudez franco. Átilio Boveti, Alfredo Gramajo Gutierrez y el potosino Victor Valdivia. Gramajo le hara la tapa de Kanchis Sorico. Noveia punene 11928), mentras gue Valdivia hará lo propio con to

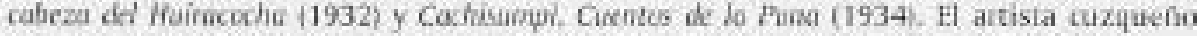
Mariano Fuentes tira le tustraria el estupendo Huilke. Coentos ded Kosho | I938) (Fig.10).

Yr adentrados en la década de 1930. podemos senalar entre los ilustradores incaistas afgentinos a Raul Manio Rossrivo, autor de cubieita e flusuaciones intesiotes para frocht nanse (1931) de Amodeo Ractolfo Sirrolii, obra coincidente en año con una de las cumbres de la ilustracion vanguardista drgentina, La edición limitada del drama ONanitoy que se pablicu entre Madrid. Paris y Puenos Aires por los Amigos del Libro de Arre, con grabados al bri en colores del escultor Pablo Curatella Manes. Orro arrista del cincel, Lais Perlotti. con estrechos vinculos en Lima y Cuzco, en sus contadas incursiones en el libro dejós tambièn la impronta prehispanista como se aprecia en las tapas de for qué estemas con fidivia (1933) de Juan Andrés Cuello Freyre, o Sabidurin de fos incas 11934 ) de Finesto Morales, ambos con huellas tiawanacotas libremente interptetadas. José Garcia Bańón hizo rarias ilustraciones para Cua Dello (1931) de Paulina Simonielo, y Pedro Heredia para su propia novela La

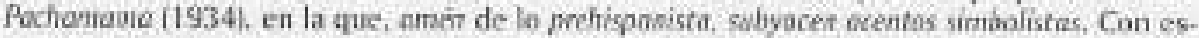
tas menciones cerramos une apretada sintesis, que deseamos sina como base para ampliar conocimientos sobre un tema que, estamos seguros, scguira brindando nuevas atalayas y descubrimicritos. 


\section{BIBLOCRAFIA}

Aceveck, Juan

1959 Malage Grentet humor grafico fimcrò. En Hisitrio de Limar $y$ atms imtas. VI Cabgaio de fisteria de tima. Maricoresa Estrada. Miguel(comp \& 33-35. Limat Universidad Nacional Mayor de San Mareos.

Ammando, Adriana, y Fustoni, Guillermo

1959 Primithismo y berencia indigena en el arte argentino de los nilos 20. En Acters. del Xir Congeso Nasinai de Arqueclogio Argentune. Diez Marin, Cristina ied. t. II, 127-131. La Plata: Editorial de la Universidad Nrcional de La Plata.

Boplione, Koccarche

2011 Vaiwenes ruptutistas. Juan Manoel Bonct, especialista en las vanguardias artisticas hispanoamericanas. In ta Diarh. Montevideo, 11 de noviembre de 2011.

Castrilko, Alfmensa

2006 Hconogratia de la Rensta Amauta Critica y gosto en lose Carlos Mariátegui - INope, 3(3): 35-44, Lima.

Fauchereau. Serge

2010 Avau-gurcles du XXce siecle Avts 5 Lithrature 7905-1930. Parisi Fammarion.

Kuon Arce, Elizabeth; Gutiérrez Vintuales, Rodrigo: Gatikrrer Bamón. y Víiesales. Graciela Maria

2009 Cazzo-Buemus Aines, Fota de wrolectesindod

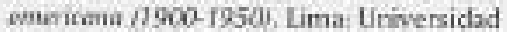
San Martin de Porres
Leguizament Poadal, Gonzala, y Gelly Cantilo. Alberto

1927 Viracocoto. Dibyjas dexaratives onsericanas. Buenos Aures: Conisión Naciotal de Bellas krtes.

Majtuf, Natalia, y Wattarden, Lais Eduarde

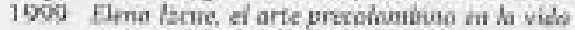
moderne. Limaz Maseo de Arte.

\section{Moreno Suntabárbara, Federico}

2004 Veinte ilustrathes spartoirs $11899-19361$. Madrie: Miaisicrio de Educacion, Cultura y Deporte

\section{Ocamapo, Esteda}

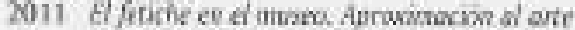
mintition_Madrid: Alianza Feitoriad

Rivera Escobar. Raul

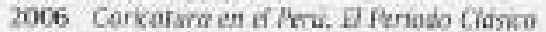
(1904-J937). Limai-Universidad de San Martin de Pottes.

Velarde, Hernán (prótogo)

1967 Moben serenet. On gevio a Lrovis del arte. Lims se.

Wye, Deborah

2002 Sobre arte y lores: uns incmoduccioa.

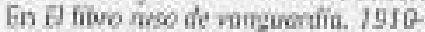
1934, Rowell, Marmit, y Wye, Deborah [coords.]. Macrid. Meseo Nacioxsal Centris de Arte Reina Solía.

Zevallos Velarde, Omas

2010 Trazos y nises las varicetoristas arequipoños. Arequips: Cuzzi Editures. 